\title{
Understanding and Managing Atopic Dermatitis in Adult Patients
}

\author{
Charles N. Ellis, MD, ${ }^{*}$ Anthony J. Mancini, MD, ${ }^{\dagger}$ Amy S. Paller, MD, ${ }^{\ddagger}$ \\ Eric L. Simpson, MD, MCR, ${ }^{\S}$ and Lawrence F. Eichenfield, MD\|"
}

\begin{abstract}
Atopic dermatitis (AD) in adults is an important dermatologic disease. Even in patients in whom the clinical presentation is mild, the burden of disease can be considerable. Relatively little has been published on adult $A D$ compared to the body of literature devoted to $A D$ in children, although adults with severe $A D$ are greatly affected by the disease. Even when $A D$ is a mild clinical disease in adults, the psychosocial and economic burden of the disease can be profound. Patients are likely to find it useful if these nondermatologic comorbidities of $A D$ are addressed by health care providers in clinical encounters. The treatment options for $A D$ in adults are the same as those for children with $A D$, with some modifications.

Semin Cutan Med Surg 31(suppl 3):S18-S22 Published by Elsevier Inc.
\end{abstract}

KEYWORDS atopic dermatitis, adults, dermatologic infections, topical anti-inflammatories, pruritus, depression

Tn about $90 \%$ of patients with atopic dermatitis (AD) in 1 childhood, the disease is diagnosed before 5 years of age. In an estimated $70 \%$ to $90 \%$ of patients, it resolves before adulthood. Thus, the disease persists into adulthood in 10\% to $30 \%$ of those diagnosed in childhood, with an estimated prevalence of $\mathrm{AD}$ in adults of $1 \%$ to $3 \% .{ }^{1}$ In the older or elderly adult population, the prevalence appears to be much lower, 2,3 but as the ESTHER study (Epidemiologische Studie zu Chancen der Verhütung, Früherkennung und optimierten

*William B. Taylor Professor of Clinical Dermatology and Associate Chair, Department of Dermatology, University of Michigan Medical School, Ann Arbor, MI

$\uparrow$ Professor of Pediatrics and Dermatology, Northwestern University Feinberg School of Medicine, and Head, Division of Pediatric Dermatology, Ann \& Robert H. Lurie Children's Hospital of Chicago, Chicago, IL.

¥Walter J. Hamlin Professor and Chair, Department of Dermatology, Professor of Pediatrics, Northwestern University Feinberg School of Medicine, Attending Physician, Ann \& Robert Lurie Children's Hospital of Chicago, Chicago, IL.

§Associate Professor of Dermatology and Director of Clinical Studies, Oregon Health and Science University, Portland, OR.

\|Professor of Clinical Pediatrics and Medicine (Dermatology), and Chief, Pediatric and Adolescent Dermatology, University of California, San Diego School of Medicine, Rady Children's Hospital, San Diego, CA

Publication of this CME article was jointly sponsored by the University of Louisville Continuing Health Sciences Education and Global Academy for Medical Education, LLC in affiliation with Skin Disease Education Foundation and is supported by an educational grant from Valeant Pharmaceuticals North America Inc.

Lawrence F. Eichenfield, MD, has served as a consultant for Anacor, Bayer, and Onset Therapeutics and as a speaker and consultant for Valeant. He has
THerapie chronischer ERkrankungen in der älteren Bevölkerung) in Germany demonstrated, AD incidence is increasing among elderly patients in industrialized countries. ${ }^{3}$

\section{Why Does AD Persist in Some Patients?}

The reason for resolution of childhood AD in most patients or for persistence of AD into adulthood in some patients has not been established, but recent research demonstrates that-

also been an investigator and consultant for Galderma, Leo Pharma as well as an investigator for Amgen, Astellas Pharma US, and Stiefel, A GSK Company.

Charles N. Ellis, MD, has served as a consultant for Galderma, Ferndale Laboratories, Medicis, and Novartis.

Anthony J. Mancini, MD, has served as a consultant for Quinnova and Valeant as well as a speaker and consultant for Galderma.

Amy S. Paller, MD, has received grant research support from Astellas.

Eric L. Simpson, MD, MCR, has served as a consultant, investigator and speaker for Galderma.

The faculty have received an honorarium from Global Academy for Medical Education for their participation in this activity. They acknowledge the editorial assistance of Joanne Still, medical writer, and Global Academy for Medical Education in the development of this continuing medical education journal article. Joanne Still has no relevant financial relationships with any commercial interests.

Address reprint requests to: Charles N. Ellis, MD, William B. Taylor Professor and Associate Chair, University of Michigan Department of Dermatology, 1500 E. Medical Center Drive, SPF 5314, Ann Arbor, MI 481095314. E-mail: cellis@umich.edu 
like the predisposition to develop $\mathrm{AD}$ itself-persistence or resolution of the disease is genetically determined.

Progress has been made within the last decade toward understanding the long-observed familial predisposition to develop $\mathrm{AD}$, advances made possible by completion of the mapping of the human genome. Loss-of-function mutations in the filaggrin gene (FLG) have been identified as being strongly associated with the development of AD. ${ }^{4}$ Subsequent studies determined that the number of FLG copies within genes can vary, with alleles encoded for 10,11 , or 12 copies of the filaggrin monomer.

Based on this understanding, Brown and colleagues ${ }^{5}$ conducted a genetic study of more than 800 children with AD and determined that the number of copies of the filaggrin monomer affect how much filaggrin protein is expressed in skin. They demonstrated that the number of copies of FLG is associated with the tendency to develop $\mathrm{AD}$, and speculated that the number of FLG copies might be associated with disease severity in a "dose-dependent" manner-fewer copies, more severe disease; more copies, less severe disease.

\section{Adult-Onset AD}

$\mathrm{AD}$ beginning in adulthood is an unusual presentation. In 2000, Bannister and Freeman ${ }^{6}$ noted that their extensive review of published articles yielded only occasional mentions of adult-onset $\mathrm{AD}$ in epidemiologic studies. In their own review of 2604 patients seen in a contact dermatitis clinic, $243(9 \%)$ had onset of AD at 20 years of age or older and had no evidence of contact dermatitis. These authors note that clinicians encounter patients with adult-onset symptoms characteristic of $\mathrm{AD}$, with cases being sometimes severe and disabling. Some of these patients are diagnosed with adultonset AD because they have a clinical presentation that suggests AD (such as flexural involvement) or because their eczematous dermatitis is accompanied by a history of allergic disease (such as asthma or hay fever) or a family history of atopic disease.

Ozkaya $^{7}$ argues that adult-onset AD may demonstrate the typical clinical pattern seen in adults who have maintained their childhood AD (which includes flexural involvement with lichenification), but also may affect the trunk and extremities, with or without flexural involvement. In a retrospective study of 376 consecutive patients with diagnoses of AD (according to the Hanifin-Rajka criteria $\left.{ }^{8}\right)$, Ozkaya ${ }^{7}$ found 63 patients (17\%) who reported onset of AD symptoms after 18 years of age (reported onset ranged from 18 to 71 years of age, with a mean of 28 years). Seven (11\%) of the 63 patients had nonflexural involvement, and, in these patients, patch testing was done to exclude detectable contact dermatitis.

Additional studies are required to characterize further, delineate, and determine criteria for the diagnosis of adultonset AD. Meanwhile, symptomatic therapy is indicated, following the same strategy that is used for patients with $\mathrm{AD}$ in whom the disease has persisted since childhood.

\section{Clinical Presentation}

The presentation of $\mathrm{AD}$ in adults may differ from that seen in children with the disease. As in childhood, the sites of involvement in adult $\mathrm{AD}$ can be anywhere on the body, but the most common areas of involvement in adults are the flexural areas of the arms and legs, the nape of the neck, and the hands. In adults, $\mathrm{AD}$ is characterized by a more lichenified, drier appearance than is usually seen in children with AD.

The psoriasiform presentation in adults might be explained by a shift in $T$ helper $\left(T_{H}\right)$ type 2 cells toward the $T_{H} 1$ phenotype. ${ }^{9}$ In a complicated study of 121 elderly patients (mean age, 69 years), Bozek and colleagues ${ }^{10}$ found that 25 (21\%) of these patients had AD with negative allergy testing and low levels of total IgE. Patients in this group tended to have $T_{H} 1$ cytokine profiles. However, sensitivity to aeroallergens, especially dust mites, often persists in adults.

Although signs and symptoms tend to be less severe in adults than in children, milder presentations can be severely problematic for adult patients, particularly when the exposed areas of the body are involved, such as the hands, neck, and face. Moreover, a subset of adults can have severe AD that is challenging to manage (Figure 1).

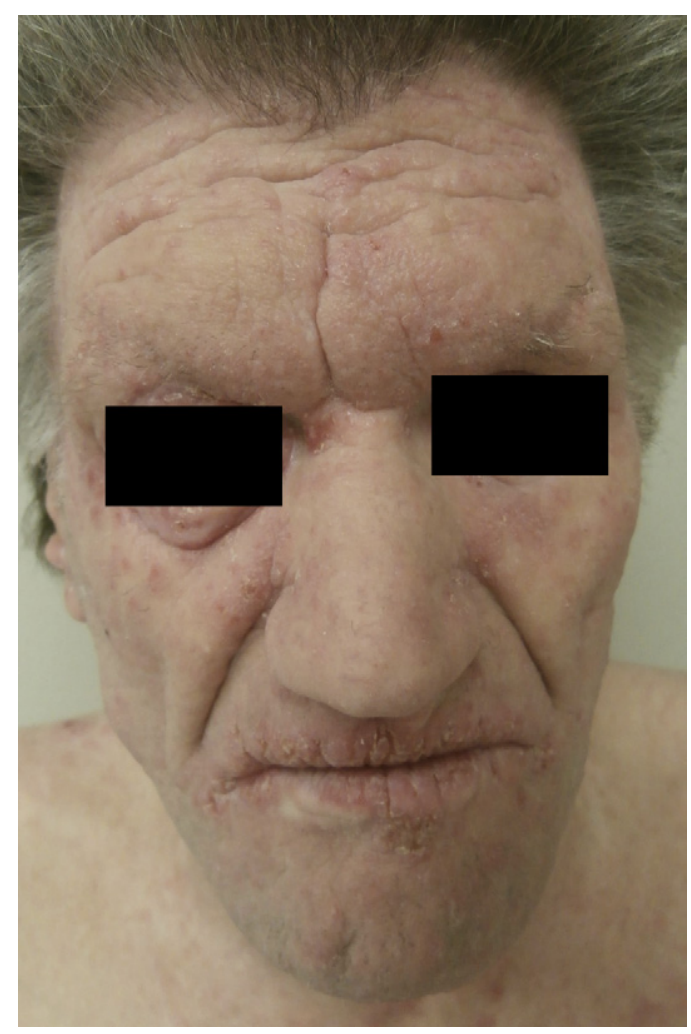

Figure 1 Atopic dermatitis in an adult. This patient has had atopic dermatitis since childhood. At the time of this clinical visit, the patient complained of pruritus and the affected areas were dry and lichenified. In such cases, bacterial colonization is likely, particularly in crusted areas such as the periorificial region shown here. Photo courtesy of Charles N. Ellis, MD. 


\section{Infectious Comorbidities in Adult AD}

Skin infection is not as common a problem in adults as in children. However, colonization with S. aureus in adult patients with $\mathrm{AD}$ is high—reported as $86 \%$ in one study ${ }^{11}$ —and is associated with the severity of disease. ${ }^{11,12}$ In addition, colonization with yeast organisms-typically, Malassezia species-is common in the head and neck areas. ${ }^{13,14}$

Eczema herpeticum (EH) can be a life-threatening or disfiguring complication of AD in adults as well as in children. The risk for this complication is increased in individuals with $\mathrm{AD}$ who have or acquire herpes simplex virus (HSV) infections. Patients with AD should take steps to avoid exposure to individuals with HSV infections, whenever possible. In addition, live vaccination for smallpox (for example, in the military) may generalize to cause eczema vaccinatum in patients with AD. Therefore, these individuals should avoid vaccinations, and those who share a residence with patients with should also avoid such vaccinations to prevent transmission to the patient with $\mathrm{AD}$. Recently, Leung and colleagues ${ }^{15}$ reported that it may be possible to determine which patients with $\mathrm{AD}$ are at risk for $\mathrm{EH}$ infections. In a study involving 64 subjects-24 with a history of this complication (ADEHpositive), 20 without such a history (ADEH-negative), and 20 nonatopic individuals - these investigators found that interferon- $\gamma$ protein production was significantly lower in the ADEH-positive subjects than in the ADEH-negative and the nonatopic control groups.

\section{Psychiatric and Psychological Comorbidities}

The literature on comorbidity in $\mathrm{AD}$ has drawn attention to concomitant depression in teenagers and adults (well-established), as well as other mental disorders, including attention-deficit/hyperactivity disorder and autism (under investigation, with emerging evidence). AD is among the group of dermatologic conditions (also including psoriasis, chronic idiopathic urticaria, and alopecia areata) in which a number of psychiatric disorders-including depression-are considered comorbidities. At least $30 \%$ of patients with dermatologic diseases have been reported to experience psychiatric disturbances and psychosocial problems. ${ }^{16}$ Even mild degrees of severity of AD may be accompanied by a psychiatric comorbidity. ${ }^{16}$

Gupta et $\mathrm{al}^{17}$ examined the relationship between pruritus and depression in 252 patients with mild to moderate psoriasis $(\mathrm{n}=77), \mathrm{AD}(\mathrm{n}=143)$, or idiopathic urticaria $(\mathrm{n}=32)$. Patients were asked to rate the severity of their pruritus on a 10-point scale devised by the investigators. They also completed psychologic rating scales to assess depression (Carroll Rating Scale for Depression), levels of anger, anxiety, and curiosity (Spielberger State-Trait Personality Inventory), and psychopathologic symptom dimensions such as depression, somatization, phobic anxiety, and paranoid ideation (Brief Symptom Inventory). In this study, the severity of pruritus in all groups of patients correlated with their levels of depression, which suggests that patients with more severe depression have a lower itch threshold, or that more severe pruritus results in higher depression scores.

Suicidal ideation is a risk in patients with depression, and Kimata ${ }^{18}$ reported that such ideation is common in patients with AD. Symptoms of major depression may be detectable during a brief office visit, but clinicians also should be alert to the possibility that patients with $\mathrm{AD}$ may be experiencing subclinical depression, characterized by less dramatic manifestations, such as a decrease in energy and interest in activities that usually bring satisfaction; individuals with subclinical depression often do not perceive themselves as feeling sad. ${ }^{16}$

In addition to frank psychiatric disturbances, $\mathrm{AD}$ is associated with psychosocial and quality-of-life impairment, a relationship that has long been recognized by clinicians and patients, and is supported by a large body of evidence in the literature.

In the landmark International Study of Life with Atopic Eczema (ISOLATE) ${ }^{19} 38 \%$ of patients with AD said that their disease affected their choice of occupation, and even those with mild to moderate $\mathrm{AD}$ reported an increase the number of sick days taken from work, as well as early retirement. Ten percent of respondents said that they believed they had experienced discrimination in their workplace; one in seven patients said that their careers had been impaired by their disease.

Psychosexual issues also were common: $58 \%$ of individuals with $\mathrm{AD}$ reported that they had decreased desire for sex, and $37 \%$ of partners of those with AD said the condition adversely affected their sexual relationship. Forty-three percent of adults with AD said that they felt awkward about having a partner see or touch their body during a flare of the disease. ${ }^{19}$

Adults with AD also avoid other activities at work and in social- and home-life. The findings in ISOLATE have been supported by other studies. ${ }^{19-23}$

Unfortunately, such issues often are inadequately addressed in clinical encounters involving adult patients. ${ }^{19,21-23}$ Only 26\% of patients in ISOLATE said that their physicians acknowledged and discussed such problems with them. ${ }^{19}$

Issues that may affect quality of life deserve more attention, and clinicians may wish to consider screening their patients with $\mathrm{AD}$ for possible clinical or subclinical psychiatric disturbances. Some patients may benefit from treatment with antidepressants or other psychotropic medications, as well as psychotherapy. Dermatologists and other clinicians who treat adult patients with $\mathrm{AD}$ also should be prepared to refer these patients to appropriate mental health professionals for evaluation and possible treatment when these issues are identified.

\section{Treatment of AD in Adults: Special Considerations}

With a few modifications, treatment options for adults with $\mathrm{AD}$ are the same as those for children with the disease (see the article by Paller et $\mathrm{al}^{24}$ on page S10 of this supplement). Because adult patients tend to have thicker, more lichenified skin, more aggressive measures may be needed to bring signs and symptoms under control. Unfortunately, only $25 \%$ of 
patients and their caregivers in the ISOLATE study feel confident that they can manage AD flares adquately, and 75\% reported that being able to have such control would be "the single most important improvement" in their quality of life. ${ }^{19}$

For mild to moderate disease in adults, higher-potency topical agents (corticosteroids or topical calcineurin inhibitors [TCIs]) are the mainstays of therapy. However, the ISOLATE survey ${ }^{19}$ revealed that $58 \%$ of patients restricted their use of topical corticosteroids because of concerns about side effects, and 66\% said they use these medications "only as a last resort." Thus, it is evident that adult patients with $\mathrm{AD}$ require education from their health providers about the realistic risks and benefits associated with the use of topical medications, including both corticosteroids and noncorticosteroid agents such as TCIs. 25,26

For adult patients with severe AD that does not respond to topical therapy, treatments include phototherapy-narrowband ultraviolet B, ultraviolet A, or both. ${ }^{27-29}$ Systemic immunosuppressants also are recognized therapeutic options in severe cases of AD. Patients in whom these agents are used-including methotrexate, azathioprine, mycophenolate mofetil, and oral calcineurin inhibitors (cyclosporine or tacrolimus) — should be followed carefully, and laboratory monitoring should be performed, as described in the article by Paller et al. ${ }^{24}$

If the skin in an adult patient with AD looks superficially infected (for example, the presence of pustules or weeping of serous fluid), bacterial cultures should be obtained and appropriate antibiotic therapy instituted. In some patients, antibiotic treatment may be helpful, even in the absence of frank infection.

Emerging understanding of the underlying pathogenesis and related molecular processes of $\mathrm{AD}$ has led to attempts to influence these mechanisms. For example, based on the discovery of the association between IgE-mediated inflammatory responses and eczematous skin signs and symptoms, and on the success of treatment to reduce IgE serum levels in patients with asthma, some have proposed the potential benefit of reducing serum IgE levels in patients with AD. A study of a small group of adults with severe AD did not yield promising results. ${ }^{30}$ However, Vigo and colleagues ${ }^{31}$ studied the effects of anti-IgE treatment on skin symptoms in two pediatric and five adult patients with $\mathrm{AD}$ who were being treated with the anti-IgE monoclonal antibody omalizumab for their asthma. In this study, the anti-IgE strategy showed substantial benefit for the skin component.

Similarly, the association of IgE-mediated activation of skin mast cells in patients with $\mathrm{AD}$, the advances in understanding of the roles of innate and adaptive immunity and skin barrier function/dysfunction, ${ }^{32}$ and the success of the leukotriene receptor antagonist montelukast in asthma and allergic rhinitis prompted the study of this agent in AD. However, a 4-week, randomized, double-blind, placebo-controlled trial of 59 adult patients with moderate to severe $\mathrm{AD}$ by Veien and colleagues ${ }^{33}$ failed to demonstrate a significant difference in efficacy between the montelukast and placebotreated groups. A subsequent 8-week, randomized, placebocontrolled trial of montelukast in adults with AD conducted by Friedmann and colleagues ${ }^{34}$ also failed to demonstrate significant benefit.

Others have examined the potential benefits in AD of topical cromolyn sodium lotion ${ }^{35}$ (some success), probiotic treatment with microorganisms such as Lactobacillus ${ }^{36}$ and oral supplementation with essential fatty acids ${ }^{37}$ (no significant benefit likely), and Chinese herbal remedies ${ }^{38}$ (not enough evidence from clinical trials to establish or rule out benefit).

\section{Conclusion}

Relatively little has been published on AD in adults compared to the literature available regarding $\mathrm{AD}$ in pediatric patients. Treatment of AD signs and symptoms in adults is not radically different from that used in younger patients; all of the options available for children may be considered for adults, and modalities - such as ultraviolet therapy—that may not be suitable for some (especially the youngest) pediatric patients may be quite effective in adults.

A substantial gap exists in treatment of $A D$ in adults with respect to the recognition and management of quality-of-life issues and psychiatric comorbidities. Addressing these comorbidities provides an important opportunity to improve the treatment of $\mathrm{AD}$ in adults.

\section{References}

1. Schmitt J, Bauer A, Meurer M: Atopic eczema in adulthood [in German]. Hautarzt 59:841-852, 2008

2. Tanei R: Atopic dermatitis in the elderly. Inflamm Allergy Drug Targets 8:398-404, 2009

3. Wolkewitz M, Rothenbacher D, Low M, et al: Lifetime prevalence of self-reported atopic diseases in a population-based sample of elderly subjects: Results of the ESTHER study. Br J Dermatol 156:693-697, 2007

4. van den Oord RA, Sheikh A: Filaggrin gene defects and risk of developing allergic sensitisation and allergic disorders: Systematic review and meta-analysis. BMJ 339:b2433, 2009

5. Brown SJ, Kroboth K, Sandilands A, et al: Intragenic copy number variation with filaggrin contributes to the risk of atopic dermatitis with a dose-dependent effect. J Invest Dermatol 132:98-104, 2012

6. Bannister MJ, Freeman S: Adult-onset dermatitis. Australas J Dermatol 41:225-228, 2000

7. Ozkaya E: Adult-onset atopic dermatitis. J Am Acad Dermatol 52:579582,2005

8. Hanifin JM, Rajka G: Diagnostic features of atopic dermatitis. Acta Derm Venereol Suppl (Stockh) 92:44-47, 1980

9. Leung DYM, Boguniewicz M, Howell MD, et al: New insights into atopic dermatitis. J Clin Invest 113:651-657, 2004

10. Bozek A, Fisher A, Filipowska B, et al: Clinical features and immunological markers of atopic dermatitis in elderly patients. Int Arch Allergy Immunol 157:372-378, 2012

11. Gilani SJ, Gonzalez M, Hussain I, et al: Staphylococcus aureus re-colonization in atopic dermatitis: Beyond the skin. Clin Exp Dermatol 30:1013,2005

12. Roll A, Cozzio A, Fischer B, et al: Microbial colonization and atopic dermatitis. Curr Opin Allergy Clin Immunol 4:373-378, 2004

13. Sandström MH, Faergemann J: Prognosis and prognostic factors in adult patients with atopic dermatitis: A long-term follow-up questionnaire study. Br J Dermatol 150:103-110, 2004

14. Sandström Falk MH, Faergemann J: Atopic dermatitis in adults: Does it disappear with age? Acta Derm Venereol 86:135-139, 2006

15. Leung DYM, Gao P-S, Grigoryev DN, et al: Human atopic dermatitis 
complicated by eczema herpeticum is associated with abnormalities in IFN- $\gamma$ response. J Allergy Clin Immunol 127:965-973, 2011

16. Fried RG, Gupta MA, Gupta AK: Depression and skin disease. Dermatol Clin 23:657-664, 2005

17. Gupta MA, Gupta AK, Schork NJ, et al: Depression modulates pruritus perception: A study of pruritus in psoriasis, atopic dermatitis, and chronic idiopathic urticaria. Psychosom Med 56:36-40, 1994

18. Kimata H: Prevalence of suicidal ideation in patients with atopic dermatitis. Suicide Life Threat Behav 36:120-124, 2006

19. Zuberbier T, Orlow SJ, Paller AS, et al: Patient perspectives on the management of atopic dermatitis. J Allergy Clin Immunol 118:226232, 2006

20. Rehal B, Armstrong AW: Health outcomes in atopic dermatitis. Dermatol Clin 30:73-86, 2012

21. Kiebert G, Sorensen SV, Revicki D, et al: Atopic dermatitis is associated with a decrement in health-related quality of life. Int J Dermatol 41: 151-158, 2002

22. Haeck IM, ten Berge O, van Velsen SGA, et al: Moderate correlation between quality of life and disease activity in adult patients with atopic dermatitis. J Eur Acad Dermatol Venereol 26:236-241, 2012

23. Kelsay K, Klinnert M, Bender B: Addressing psychosocial aspects of atopic dermatitis. Immunol Allergy Clin North Am 30:385-396, 2010

24. Paller AS, Eichenfield LE, Ellis C, et al: Treatment strategies for atopic dermatitis: Optimizing the available therapeutic options. Semin Cutan Med 31:S10-S17, 2012 (suppl)

25. Callen J, Chamlin S, Eichenfield LF, et al: A systematic review of the safety of topical therapies for atopic dermatitis. Br J Dermatol 156:203221, 2007

26. Gisondi P, Ellis CN, Girolomoni G: Pimecrolimus in dermatology: Atopic dermatitis and beyond. Int J Clin Pract 59:969-974, 2005

27. Reynolds NJ, Franklin V, Gray JC, et al: Narrow-band ultraviolet B and broad-band ultraviolet A phototherapy in adult atopic eczema: A randomised controlled trial. Lancet 357:2012-2016, 2001
28. Majoie IM, Oldhoff JM, van Weelden $\mathrm{H}$, et al: Narrowband ultraviolet B and medium-dose ultraviolet $\mathrm{Al}$ are equally effective in the treatment of moderate to severe atopic dermatitis. J Am Acad Dermatol 60:77-84, 2009

29. Gambichler T, Othlinghaus N, Tomi NS, et al: Medium-dose ultraviolet (UV) Al vs. narrowband UVB phototherapy in atopic eczema: A randomized crossover study. Br J Dermatol 160:652-658, 2009

30. Krathen RA, Hsu S: Failure of omalizumab for treatment of severe adult atopic dermatitis. J Am Acad Dermatol 53:338-340, 2005

31. Vigo PG, Girgis KR, Pfuetze BL, et al: Efficacy of anti-IgE therapy in patients with atopic dermatitis. J Am Acad Dermatol 55:168-170, 2006

32. Jung T, Stingl G: Atopic dermatitis: Therapeutic concepts evolving from new pathophysiologic insights. J Allergy Clin Immunol 122: 1074-1081, 2008

33. Veien NK, Busch-Sorensen M, Stausbøl-Gron B: Montelukast treatment of moderate to severe atopic dermatitis in adults: A randomized, double-blind, placebo-controlled trial. J Am Acad Dermatol 53:147-149, 2005

34. Friedmann PS, Palmer R, Tan E, et al: A double-blind, placebo-controlled trial of montelukast in adult atopic eczema. Clin Exp Allergy 37:1536-1540, 2007

35. Stainer R, Matthews S, Arshad SH, et al: Efficacy and acceptability of a new topical skin lotion of sodium cromoglicate (Altoderm) in atopic dermatitis in children aged 2-12 years: A double-blind, randomized, placebo-controlled trial. Br J Dermatol 152:334-341, 2005

36. van der Aa LB, Heymans HS, van Aalderen WM, et al: Probiotics and prebiotics in atopic dermatitis: Review of the theoretical background and clinical evidence. Pediatr Allergy Immunol 21:e355-e367, 2010

37. van Gool CJ, Zeegers MP, Thijs C: Oral essential fatty acid supplementation in atopic dermatitis-a meta-analysis of placebo-controlled trials. Br J Dermatol 150:728-740, 2004

38. Zhang W, Leonard T, Bath-Hextall F, et al: Chinese herbal medicine for atopic eczema. Cochrane Database Syst Rev CD002291, 2004 